UMN-TH-1334/95

LBL-37019

UCB-95/109

hep-ph/yymmddd

April 1995

\title{
Preserving Flat Directions During Inflation \&
}

\author{
Mary K. Gaillard \\ Department of Physics and Theoretical Physics Group, Lawrence Berkeley Laboratory \\ University of California, Berkeley, California, 94720 \\ Hitoshi Murayamal \\ Theoretical Physics Group, Lawrence Berkeley Laboratory \\ University of California, Berkeley, CA 94720, USA \\ and \\ Keith A. Olive \\ School of Physics and Astronomy, University of Minnesota \\ Minneapolis, MN 55455, USA
}

\begin{abstract}
Supersymmetry is generally broken by the non-vanishing vacuum energy density present during inflation. In supergravity models, such a source of supersymmetry breaking typically makes a contribution to scalar masses of order $\tilde{m}^{2} \sim H^{2}$, where $H^{2} \sim V / M_{P}^{2}$ is the Hubble parameter during inflation. We show that in supergravity models which possess a Heisenberg symmetry, supersymmetry breaking makes no contribution to scalar masses, leaving supersymmetric flat directions flat at tree-level. One-loop corrections in general lift the flat directions, but naturally give small negative squared masses $\sim-g^{2} H^{2} /(4 \pi)^{2}$ for all flat directions that do not involve the stop. No-scale supergravity of the SU(N,1) type and the untwisted sectors from orbifold compactifications are special cases of this general set of models. We point out the importance of the preservation of flat directions for baryogenesis.

*This work was supported in part by DoE grants DE-FG02-94ER-40823 and DE-AC03-76SF00098, and by NSF grants AST-91-20005 and PHY-90-21139.

†On leave of absence from Department of Physics, Tohoku University, Sendai, 980 Japan
\end{abstract}




\section{Disclaimer}

This document was prepared as an account of work sponsored by the United States Government. While this document is believed to contain correct information, neither the United States Government nor any agency thereof, nor The Regents of the University of California, nor any of their employees, makes any warranty, express or implied, or assumes any legal liability or responsibility for the accuracy, completeness, or usefulness of any information, apparatus, product, or process disclosed, or represents that its use would not infringe privately owned rights. Reference herein to any specific commercial products process, or service by its trade name, trademark, manufacturer, or otherwise, does not necessarily constitute or imply its endorsement, recommendation, or favoring by the United States Government or any agency thereof, or The Regents of the University of California. The views and opinions of authors expressed herein do not necessarily state or reflect those of the United States Government or any agency thereof, or The Regents of the University of California.

Lawrence Berkeley Laboratory is an equal opportunity employer. 
One of the more robust mechanisms for the generation of a cosmological baryon asymmetry is the decay of the coherent scalar field oscillations along flat directions in supersymmetric theories, commonly referred to as the Affleck-Dine (AD) mechanism [1]. It is well known that in the supersymmetric standard model and in supersymmetric grand unified theories, there are directions in field space in which both the $F$ - and $D$-terms of the scalar potential vanish identically. Such a direction was explicitly constructed in the context of SU(5) in [1] and generalized to larger guts as well [2]. Supersymmetry breaking spoils the flatness, and one expects the generation of soft scalar masses for all scalar fields of order of the supersymmetry breaking scale, $\tilde{m}$. A baryon asymmetry can be generated if at some early stage in the evolution of the Universe, the fields along the flat direction obtain large vacuum expectation values and can be associated with a baryon number violating operator. Subsequently, when the expansion rate, given by the Hubble parameter, $H$, is of order $\tilde{m}$, coherent scalar field oscillations along the flat direction carrying a baryon number begin. The decay of the scalar field oscillations results in a final baryon asymmetry [1, 3].

The AD scenario outlined above is normally implemented in the context of inflation 4 . During inflation, scalar fields with masses, $m<H$, are driven by quantum fluctuations to large vacuum values which become the source of the scalar field oscillations once inflation is over. To avoid the washout of the baryon asymmetry by electroweak sphaleron interactions [5], the scenario works most easily in the framework of a gut larger than minimal SU(5) in which $B-L$ is violated, though it is possible that the asymmetry can be preserved even when $B-L$ is not violated [6]. Indeed, it is possible to implement the AD scenario for baryogenesis without any additional baryon number violation beyond that in the standard model so long as lepton number is violated [7]. Here, flat directions associated with lepton number violating operators can be used to generate a lepton asymmetry which is subsequently converted into a baryon asymmetry by sphaleron interactions.

Recently, it has been argued that the simple picture of driving scalar fields to large vacuum values along flat directions during inflation is dramatically altered in the context of supergravity [8]. During inflation, the Universe is dominated by the vacuum energy density, $V \sim H^{2} M_{P}^{2}$. The presence of a non-vanishing and positive vacuum energy density indicates that supergravity is broken and soft masses of order of the gravitino mass $\sim H$ are generated [9]. A similar observation was made in [10] where it was noted that other flat directions, associated with moduli, also receive large contributions to their masses from a non-zero 
vacuum energy density during inflation. There it was argued that in such cases there may be no moduli problem (a.k.a. Polonyi problem [11]) involving excess entropy production. However this solution would require that the expectation value of the moduli are unchanged during and after inflation, an unlikely circumstance. The contribution to scalar masses can be easily understood by considering for example, the scalar potential in a supergravity model described by a Kähler potential $G$ [12],

$$
V=e^{G}\left[G_{i}\left(G^{-1}\right)_{j}^{i} G^{j}-3\right]
$$

where $G_{i}=\partial G / \partial \phi^{i}$ and $G^{i}=\partial G / \partial \phi_{i}^{*}$. A positive vacuum energy density, $V>0$, needed for inflation, requires that $e^{G} G_{i} \neq 0$, thus breaking supergravity, and implies that the gravitino mass, $m_{3 / 2}=e^{G / 2} \neq 0$. Typically, scalar squared masses will pick up a contribution of order $m_{3 / 2}^{2} \sim V / M_{P}^{2} \sim H^{2}$, thus lifting the flat directions and potentially preventing the realization of the AD scenario as argued in $[8]$. Below we use reduced Planck units: $M_{P} / \sqrt{8 \pi}=1$.

To see this, let us consider a minimal supergravity model whose Kähler potential is defined by

$$
G=z z^{*}+\phi_{i}^{*} \phi^{i}+\ln |\bar{W}(z)+W(\phi)|^{2}
$$

where $z$ is a Polonyi-like field [13] needed to break supergravity, and we denote the scalar components of the usual matter chiral supermultiplets by $\phi^{i}$. $W$ and $\bar{W}$ are the superpotentials of $\phi^{i}$ and $z$ respectively. In this case, the scalar potential becomes

$$
V=e^{z z^{*}+\phi_{i}^{*} \phi^{i}}\left[\left|\bar{W}_{z}+z^{*}(\bar{W}+W)\right|^{2}+\left|W_{\phi^{i}}+\phi_{i}^{*}(\bar{W}+W)\right|^{2}-3|(\bar{W}+W)|^{2}\right]
$$

Included in the above expression for $V$, one finds a mass term for the matter fields $\phi^{i}$, $e^{G} \phi_{i}^{*} \phi^{i}=m_{3 / 2}^{2} \phi_{i}^{*} \phi^{i}$. If $z$ breaks supergravity this term is non-vanishing. As it applies to all scalar fields (in the matter sector), all flat directions are lifted by it as well.

Another instructive way to see the lifting of flat directions is to examine the expression (see, for example [14]) for the trace of the scalar squared mass matrix (we ignore $D$-terms assuming the inflaton is a gauge singlet),

$$
\operatorname{Tr} \mathcal{M}_{0}^{2}=2 \operatorname{Tr} \mathcal{M}_{1 / 2}^{2}+2(N-1) V+2 m_{3 / 2}^{2}\left[(N-3)-G_{i}\left(G^{-1}\right)_{j}^{i} R_{k}^{j}\left(G^{-1}\right)_{l}^{k} G^{l}\right]
$$

where $(N-1)$ is the total number of chiral multiplets $\phi^{i}, R_{i}^{j}=(\ln \operatorname{det} G)_{i}^{j}$ is the Kähler Ricci tensor, and $\operatorname{Tr} \mathcal{M}_{1 / 2}^{2}$ is the flat space value of the trace of the chiral fermion squared 
mass matrix. In the minimal supergravity model described by eq. (2), $G_{i}^{j}=\delta_{i}^{j}$ and $R_{i}^{j}=0$. Thus the traces give

$$
\operatorname{Tr}\left(\mathcal{M}_{0}^{2}-2 \mathcal{M}_{1 / 2}^{2}\right)_{\phi}+m_{A}^{2}+m_{B}^{2}-2 m_{\chi}^{2}=-4 m_{3 / 2}^{2}+2(N-1)\left(m_{3 / 2}^{2}+V\right)
$$

guaranteeing a splitting among each of the chiral multiplets $\phi$ by an amount $m_{3 / 2}^{2}$ (neglecting space-time curvature contributions to fermion masses). In (5), $m_{A}$ and $m_{B}$ are the masses of the scalar components of $z, \chi$ is its fermion component (i.e., the goldstino: $m_{\chi}=2 m_{3 / 2}$ at the ground state), and their masses satisfy $m_{A}^{2}+m_{B}^{2}-2 m_{\chi}^{2}=-4 m_{3 / 2}^{2}$. As shown below, a more careful definition of scalar "masses" is required when we consider non-minimal supergravity models.

The above arguments can be generalized to supergravity models with non-minimal Kähler potentials. By and large the same conclusions hold as claimed in [\&]. However as we will show below, there is a wide class of models with considerable phenomenological interest in which flat directions are not lifted by supergravity breaking. Indeed, the flatness is ensured by a "Heisenberg symmetry" [15] of the kinetic function in this class of models.

A special class of these models are no-scale supergravity models, that were first introduced in [16] and have the remarkable property that the gravitino mass is undetermined at the tree level despite the fact that supergravity is broken. No-scale supergravity has been used heavily in constructing supergravity models in which all mass scales below the Planck scale are determined radiatively [17, [18]. Indeed, the decoupling of the gravitino mass from global supersymmetry breaking in the observable sector was used to derive models with a gravitino mass at the Planck scale for the case [19 $g \equiv 0$ and within a few orders of that scale for the case [27] $W_{i} \equiv 0$, with phenomenologically acceptable supersymmetry breaking in the matter sector. A large gravitino mass allows a sufficiently early decay of the gravitino, thus avoiding 20 potential cosmological problems associated with the gravitino [21. In this context, the moduli problems can also be resolved [22. Many inflationary models have also been considered in the context of no-scale supergravity [23]. These models emerge naturally in torus [24] or, for the untwisted sector, orbifold [25] compactifications of the heterotic string.

We will consider the possibility that the inflaton is one of the $\phi^{i}$ or is a "hidden sector" field. Thus we introduce the set of chiral fields $z, \phi^{i}, y^{a}$, with $y^{a}$ in the hidden sector, and 
define the "Heisenberg symmetry" as follows [15],

$$
\delta z=\epsilon_{i}^{*} \phi^{i}, \quad \delta \phi^{i}=\epsilon^{i}, \quad \delta y^{a}=0,
$$

where $\epsilon^{i}$ are complex parameters and $\epsilon_{i}^{*}$ their complex conjugates. The invariants under this symmetry are the $y^{a}$ and the combination

$$
\eta \equiv z+z^{*}-\phi_{i}^{*} \phi^{i}
$$

Let us assume this is a symmetry of the kinetic function in the Kähler potential. We also require that the field $z$ does not have a coupling in the superpotential. Then the most general Kähler potential becomes

$$
G=f(\eta)+\ln |W(\phi)|^{2}+g(y),
$$

where the superpotential $W$ is a holomorphic function of $\phi^{i}$ only.

To analyze the resulting theory, we first look at the kinetic terms of the chiral scalars. In terms of the original fields $z$ and $\phi^{i}$, the kinetic terms are written as

$$
K=f^{\prime \prime}|\partial z|^{2}+f^{\prime \prime} \phi^{i} \partial z \partial \phi_{i}^{*}+f^{\prime \prime} \phi_{j}^{*} \partial z^{*} \partial \phi^{j}+\left(f^{\prime \prime} \phi_{j}^{*} \phi^{i}-f^{\prime} \delta_{j}^{i}\right) \partial \phi^{j} \partial \phi_{i}^{*}
$$

where I denotes differentiation with respect to $\eta$. To "diagonalize" the kinetic term so that chiral scalars do not have mixing kinetic terms, we rewrite the kinetic terms in terms of $\eta$ defined in Eq. (7) and a $U(1)$ current 26, 27, 28]

$$
I_{\mu}=i\left(\partial_{\mu} z-\partial_{\mu} z^{*}\right)-i\left(\phi^{i} \partial_{\mu} \phi_{i}^{*}-\phi_{i}^{*} \partial_{\mu} \phi^{i}\right)
$$

and they read as

$$
K=f^{\prime \prime}\left[(\partial \eta)^{2}+\left(I_{\mu}\right)^{2}\right]-f^{\prime}\left|\partial \phi^{i}\right|^{2} .
$$

Therefore, we regard $\eta$ and $\phi^{i}$ as independent degrees of freedom rather than $z$ and $\phi^{i}$.

We are now at the stage to write down the scalar potential. Following the general definition (11) and matrix inversion [29],

$$
V=e^{f(\eta)+g(y)}\left[\left(\frac{f^{\prime 2}}{f^{\prime \prime}}-3\right)|W|^{2}-\frac{1}{f^{\prime}}\left|W_{i}\right|^{2}+g_{a}\left(g^{-1}\right)_{b}^{a} g^{b}\right] .
$$

It is important to notice that the cross term $\left|\phi_{i}^{*} W\right|^{2}$ has disappeared in the scalar potential. Because of the absence of the cross term, flat directions remain flat even during the inflation. 
A detailed discussion of the dynamics of $\eta$ field during inflation can be found in Ref. [29] for a specific choice of $f=(3 / 8) \ln \eta+\eta^{2}$. The no-scale model corresponds to $f=-3 \ln \eta$, $f^{\prime 2}=3 f^{\prime \prime}$ and the first term in (12) vanishes. The potential then takes the form

$$
V=e^{g}\left[e^{\frac{2}{3} f}\left|W_{i}\right|^{2}+e^{f} g_{a}\left(g^{-1}\right)_{b}^{a} g^{b}\right],
$$

which is positive definite. The requirement that the vacuum energy vanishes implies $\left\langle W_{i}\right\rangle=\left\langle g_{a}\right\rangle$ at the minimum. As a consequence $\eta$ is undetermined and so is the gravitino mass $m_{3 / 2}(\eta)$.

The easiest way to see that the Lagrangian (12) preserves the flat direction is to look at the equation of motion of the fields $\phi^{i}$. In the inflationary universe, it reads as

$$
\ddot{\phi}^{i}+3 H \dot{\phi}^{i}+\Gamma_{j \eta}^{i} \dot{\phi}^{j} \dot{\eta}-\frac{e^{f+g}}{f^{\prime}}\left[\left(\frac{f^{\prime 2}}{f^{\prime \prime}}-3\right) W_{i} W^{*}-\frac{1}{f^{\prime}} W_{i j}\left(W_{j}\right)^{*}\right]=0,
$$

where the connection $\Gamma_{j \eta}^{i}$ is defined below. Since the flat direction is characterized by $W_{i}=0$, $W_{i j}=0$, it is easy to see that a constant $\phi^{i}$ satisfies the equation of motion for any values of $\phi^{i}$ along the flat direction.

It is probably also useful to look at the effective mass term for the quantum fluctuations during inflation. For definiteness, we restrict ourselves to the no-scale case in the following discussion. The usual procedure of the covariant expansion around the background fields is as follows. We start from the general action

$$
I[\phi]=\int d^{4} x \sqrt{-g}\left[\frac{1}{2} g^{\mu \nu} G_{\alpha \beta}(\phi) \partial_{\mu} \phi^{\alpha} \partial_{\nu} \phi^{\beta}-V(\phi)\right],
$$

and expand the fields as

$$
\phi^{\alpha}=\phi_{0}^{\alpha}+\xi^{\alpha}-\frac{1}{2} \Gamma_{\beta \gamma}^{\alpha} \xi^{\beta} \xi^{\gamma}+\mathcal{O}\left(\xi^{3}\right) .
$$

Here and below, the indices $\alpha, \beta, \ldots$ refer generically to $\eta, \phi^{i}, \phi_{i}^{*}$, and the Christoffel symbol $\Gamma_{\beta \gamma}^{\alpha}$ is derived from the metric $G_{\alpha \beta}(\phi)$ on the field space. We obtain

$$
\begin{aligned}
I[\phi] & =I\left[\phi_{0}\right]+\int d^{4} x \sqrt{-g}\left[g^{\mu \nu} G_{\alpha \beta} \partial_{\mu} \phi_{0}^{\alpha} D_{\nu} \xi^{\beta}-\partial_{\alpha} V \xi^{\alpha}\right] \\
& +\frac{1}{2} \int d^{4} x \sqrt{-g}\left[g^{\mu \nu} G_{\alpha \beta} D_{\mu} \xi^{\alpha} D_{\nu} \xi^{\beta}+R_{\alpha \gamma \delta \beta} \xi^{\gamma} \xi^{\delta} g^{\mu \nu} \partial_{\mu} \phi_{0}^{\alpha} \partial_{\nu} \phi_{0}^{\beta}-\left(D_{\alpha} \partial_{\beta} V\right) \xi^{\alpha} \xi^{\beta}\right] \\
& +\mathcal{O}\left(\xi^{3}\right) .
\end{aligned}
$$


The covariant derivative is defined by $D_{\alpha} \xi^{\beta}=\partial_{\alpha} \xi^{\beta}+\Gamma_{\alpha \gamma}^{\beta} \xi^{\gamma}$. The last term contains the effective term mass term for the fluctuation $\xi^{\alpha}$,

$$
\left(m^{2}\right)_{\alpha \beta}=D_{\alpha} \partial_{\beta} V=\partial_{\alpha} \partial_{\beta} V-\Gamma_{\alpha \beta}^{\kappa} \partial_{\kappa} V
$$

and its trace gives the field reparametrization invariant result (丑), with $R_{k}^{j}=\frac{1}{3}(N+1) G_{k}^{j}$ in the no-scale case. We now look at the fluctuation $\xi^{i}$ of the $\phi^{i}$ fields. In our case, however, we assume that the $\eta$ field is constant during inflation and do not use the equation of motion for the $\eta$ field. If we used the equation of motion, the $\eta$ field would roll down the potential, and the potential identically vanishes in the limit $\eta \rightarrow \infty$. It is usually assumed that higher order corrections to the potential stops this run-away behavior, and $\eta$ will be fixed at some point. How this may explicitly be realized will be discussed below. Following this standard approach and regarding the $\eta$ field as a constant, we do not allow a fluctuation for $\eta$. This in turn means $\xi^{\eta}-\Gamma_{i j}^{\eta} \xi^{i} \xi^{j} / 2=0+O\left(\xi^{3}\right)$. Then the linear term in the $\xi^{\eta}$ does not vanish in $I[\phi]$, but precisely cancels the second term $\Gamma_{i j}^{\eta} \partial_{\eta} V$ in $\left(m^{2}\right)_{i j}$. The effective mass term is hence just $\partial_{i} \partial_{j} V$. Then it should be clear from the explicit form of the potential $V$ that there is no additional mass term to the flat direction even during inflation. $f$

A natural question arises as to whether higher order corrections modify the form of the Kähler potential, thereby lifting the flat direction. Indeed, only the kinetic energy part of our Kähler potential respects the Heisenberg symmetry, which is broken by gauge couplings as well as by the superpotential. However, an explicit one-loop calculation shows that the gravitational interactions preserve the Heisenberg symmetry [15. Therefore, the only possible contribution to the mass of the flat directions come from either gauge interactions or superpotential couplings that contribute to the renormalized Kähler potential. Using the general results [14, 30] for the one-loop corrected supergravity lagrangian, we obtain, assuming that inflation is driven by an $F$-term rather than a $D$-term (the result is similar if $\langle D\rangle \neq 0)$ :

$$
\begin{aligned}
\left(m^{2}\right)_{i}^{j}= & \frac{\ln \left(\Lambda^{2} / \mu^{2}\right)}{32 \pi^{2}}\left\{h_{i k l} h^{* j k l}\left[\alpha\langle V\rangle+m_{3 / 2}^{2}\left(5 \frac{f^{\prime 2}}{f^{\prime \prime}}+2 \frac{f^{\prime} f^{\prime \prime \prime}}{f^{\prime \prime 2}}-10-\frac{f^{\prime 4}}{f^{\prime \prime 2}}\right)\right]\right. \\
& \left.-4 \delta_{i}^{j} g_{a}^{2} C_{2}^{a}\left(R_{i}\right)\left[\beta\langle V\rangle+m_{3 / 2}^{2}\left(\frac{f^{\prime} f^{\prime \prime \prime}}{f^{\prime \prime 2}}-2\right)\right]\right\},
\end{aligned}
$$

*For a more general choice of $f(\eta)$ as in 29], the potential may not have this run-away behavior for $\eta$. In this case, $\eta$ will settle to its minimum during inflation, and $V_{\eta}$ vanishes there. Therefore, the effective mass term is also just $\partial_{i} \partial_{j} V$ in the more general case as well. 
where the vacuum energy $\langle V\rangle$ and the gravitino mass $m_{3 / 2}$ are their values during inflation. $\mu \geq\langle V\rangle$ is the appropriate infrared cut-off in the loop integral, and $\Lambda$ is the cutoff scale below which the effective supergravity Lagrangian given by the Kähler potential eq. (8) is valid; $\Lambda=1$ in many models but can be lower if the inflaton is a composite field. The $h$ 's are Yukawa coupling constants $g_{a}, C_{2}^{a}\left(R_{i}\right)$ are the coupling constant and matter Casimir for the factor gauge group $G_{a}$, and the parameter $\alpha, \beta$, are model dependent. In the no-scale case $f=-3 \ln \eta$, the result (19) reduces tof

$$
\left(m^{2}\right)_{i}^{j}=\frac{\ln \left(\Lambda^{2} / \mu^{2}\right)}{32 \pi^{2}}\langle V\rangle\left[\alpha h_{i k l} h^{* j k l}-4 \beta \delta_{i}^{j} g_{a}^{2} C_{2}^{a}\left(R_{i}\right)\right]
$$

If the inflaton is one of the $\phi^{i}\left(\phi_{0} \neq 0\right), \alpha=\beta=\frac{2}{3}$. If the inflaton is in the hidden sector $\left(y_{a} \neq 0\right), \alpha<0, \beta=1$ if the inflaton is not the dilaton $s:\langle s\rangle=g^{-2}$. If the inflaton is the standard string dilaton, $\beta=\alpha=-1$. In all but the last case the masses are negative if gauge couplings dominate Yukawa couplings. In the following we assume the inflaton is not the dilaton.

There are several interesting points in the above formulae. First of all, higher order effects are always suppressed by the typical one-loop factor $1 /(4 \pi)^{2}$. The vacuum energy is related to the expansion rate by $H^{2}=(1 / 3)\langle V\rangle$, and hence the typical mass of the flat directions is $m^{2} \simeq 10^{-2} H^{2}$ during inflation. While these one-loop masses are small enough to generate classical fields on large scales during inflation, they are too large to allow sufficient growth due to quantum fluctuations in order to generate a sizeable baryon asymmetry. During inflation, quantum fluctuations in $\left\langle\phi^{2}\right\rangle$ begin to grow in time as $H^{3} t / 4 \pi^{2}$ [31 up to a limiting value given by $\left\langle\phi^{2}\right\rangle=3 H^{4} / 8 \pi^{2} m^{2}$. During inflation, the low momentum modes of these fluctuations will be indistinguishable from a classical field with an amplitude $\phi_{0} \simeq \sqrt{\left\langle\phi^{2}\right\rangle}$. In the $(\mathrm{AD})$ mechanism for baryogenesis with inflation, the baryon asymmetry produced is [4]

$$
\frac{n_{B}}{s} \sim \frac{\epsilon \phi_{0}^{4} m_{I}^{3 / 2}}{M_{X}{ }^{2} M_{P}{ }^{5 / 2} \tilde{m}} \lesssim 10^{12}\left(\frac{\phi_{0}}{M_{P}}\right)^{4}
$$

where $m_{I} \sim 10^{-7} M_{P}$ is the inflaton mass, $M_{X} \sim 10^{-3} M_{P}$ is the scale associated with the baryon number violation, $\tilde{m} \sim 10^{-16}$ is the susy breaking scale when $V=0$, i.e. after inflation, and $\epsilon$ is a measure of the $\mathrm{CP}$ violation and in this case can easily be $\mathrm{O}(1)$. If $\phi_{0}{ }^{2}$

\footnotetext{
${ }^{\dagger}$ In the context of string theory, Eq. (20) is valid in more realistic multi-moduli no-scale models that describe the untwisted sectors from orbifold compactifications.
} 
were given by the maximum value of the fluctuations generated by inflation, then ${\phi_{0}}^{2} \sim$ $6 H^{2} \sim 10^{-13} M_{P}^{2}$ and too small an asymmetry would result. However, as we have seen in eq. (20), for all scalar matter fields aside from stops, the contribution to the mass squared is negative as the Yukawa couplings are smaller than the gauge couplings. Thus any flat direction not involving stops, will have a negative contribution at one-loop without an ad hoc choice of the parameters. Now, even though fluctuations will begin the growth of $\phi_{0}$, the classical equations of motion soon take over. The classical equations of motion drive $\phi_{0}$ as $\left(-m^{2}\right) t$ which is smaller than the quantum growth only for $H t<H^{4} / m^{4}$. Then for $H t>H^{2} /\left(-m^{2}\right)$, the classical growth of $\phi_{0}$, becomes nonlinear $\sim H e^{-m^{2} t / 3 H}$, and $\phi_{0}$ will run off to its minimum determined by the one-loop corrections to $\phi^{4}$, which are again of order $V$. An explicit one-loop calculation [14, 30] shows that the effective potential along the flat direction has a form

$$
V_{e f f} \sim \frac{g^{2}}{(4 \pi)^{2}}\langle V\rangle\left(-2 \phi^{2} \log \left(\frac{\Lambda^{2}}{g^{2} \phi^{2}}\right)+\phi^{2}\right)+\mathcal{O}(\langle V\rangle)^{2},
$$

where $\Lambda$ is the cutoff of the effective supergravity theory, and has a minimum around $\phi \simeq$ $0.5 \Lambda$. Also this is consistent because this effective potential is only of order $-\langle V\rangle g^{2} /(4 \pi)^{2}$ and is a small correction to the inflaton energy density which drives the inflation. Thus, $\phi_{0} \sim M_{P}$ will be generated and in this case the subsequent sfermion oscillation will dominate the energy density and a baryon asymmetry will result which is independent of inflationary parameters as originally discussed in [1, 3] and will produce $n_{B} / s \sim O(1)$.

Finally, as noted above, in order to realize the scenario presented here in the no-scale case, we need a mechanism to stabilize the $\eta$-field. In no-scale models, typically $\langle V\rangle=$ $0, m_{3 / 2}^{2} \neq 0$ at the ground state. Therefore it is very plausible that there is a solution with $\langle V\rangle \sim \ln \left(\Lambda^{2} / m_{3 / 2}^{2}\right) m_{3 / 2}^{4} / 32 \pi^{2} \ll m_{3 / 2}^{2}$ during inflation. Under this assumption, the one loop corrections [14] give a contribution

$$
\Delta V=-\frac{1}{32 \pi^{2}}\left[\alpha m_{3 / 2}^{4} \ln \left(\Lambda^{2} / m_{3 / 2}^{2}\right)+O\left(V^{2}\right)\right],
$$

\footnotetext{
$\ddagger$ We do not need to choose a particular set of parameters because the flat direction is preserved at treelevel, and the one-loop correction is a small perturbation. In models where the flat directions are lifted at tree level, one has to add by hand tree-level terms like $\delta G=c|\phi|^{2}|\psi|^{2}$ to the Kähler potential, and impose [8] $c<0$, where $\psi$ is the inflaton, in order to generate a total negative squared mass (in minimal supergravity, the tree level squared masses that are generated during inflation are positive as seen in eq.(5)). However, this is an $\mathrm{O}(1)$ effect, and one has to be careful not to spoil the positivity of the potential and the kinetic terms. For instance, one has to impose $-1<c<-2 / 3$ for the case of the inflaton potential in [32].
} 
with $m_{3 / 2}^{2} \propto e^{f(\eta)}$, and $\alpha$ is model-dependent. If $\phi_{0}$ is the inflaton, $\alpha=14$, for a hidden sector inflaton $y_{o}, \alpha=13-21 . \beta$ For a hidden sector inflaton, $V \propto e^{f(\eta)}$ and the requirements that $V+\Delta V>0, m_{\eta}^{2}>0$, give give the condition $3 / 2>\ln \left(\Lambda^{2} / m_{3 / 2}^{2}\right)>1$ which could be considered as a fine tuning condition. Actually, these values correspond to results found (see, e.g. [18, 27]) in models where the symmetry breaking potential is generated by condensation at a scale $\Lambda$. For an inflaton $\phi_{0}, V \propto e^{f(\eta) / 3}$, and the $m_{\eta}^{2}$ mass is always positive; positivity of the one-loop corrected potential requires only $\ln \left(\Lambda^{2} / m_{3 / 2}^{2}\right)>5 / 3$ Once the positivity requirements are satisfied, we obtain $m_{\eta}^{2} \sim\langle V\rangle$, which is sufficient to assure that inflation can occur in the false vacuum.

In summary, we have shown that although it is a relatively general property that flat directions receive tree-level masses during inflation, this conclusion does not apply to the a class of models which possess a Heisenberg symmetry. This class contains the phenomenologically interesting no-scale supergravity models as well the forms of supergravity expected from string theory truncations. We have also shown that although flat directions remain flat at the tree-level in this class of models, one-loop corrections upset the flatness and we expect that for all flat directions which do not involve stops, a negative mass squared is generated reulting in large expectation values along the flat directions, leading, in turn, to (AD) baryogenesis along the lines originally suggested.

\section{Acknowledgements}

We would like to thank Bruce Campbell for helpful conversations. This work was supported in part by the Director, Office of Energy Research, Office of High Energy and Nuclear Physics, Division of High Energy Physics of the U.S. Department of Energy under Contracts DE-FG02-94ER-40823 and DE-AC03-76SF00098, by NSF grants AST-91-20005 and PHY90-21139.

\footnotetext{
${ }^{\S}$ If there are $N_{h}$ additional hidden sector scalars with masses of order $m_{3 / 2}, \alpha \rightarrow \alpha+N_{h}$; here we set $N_{h}=0$.
} 


\section{References}

[1] I. Affleck and M. Dine, Nucl. Phys. B249 (1985) 361.

[2] D. Morgan, Nucl. Phys. B364 (1991) 401; B. Campbell, J. Ellis, D.V. Nanopoulos, and K. A. Olive, Mod. Phys. Lett. A1 (1986) 389.

[3] A.D. Linde, Phys. Lett. B160 (1985) 243.

[4] J. Ellis, K. Enqvist, D.V. Nanopoulos, and K.A. Olive, Phys. Lett. B191(1987) 343.

[5] P. Arnold and L. McLerran, Phys. Rev. D36 (1987) 581; D37 (1988) 1020; L. Carson, X. Li, L. McLerran, R. Wang, Phys. Rev. D42 (1990) 2127.

[6] S. Davidson, H. Murayama, and K.A. Olive, Phys. Lett. B328 (1994) 354; S. Davidson, K. Kainulainen, and K.A. Olive, Phys. Lett. B335 (1994) 339.

[7] B. Campbell, S. Davidson, and K.A. Olive, Phys. Lett. B303 (1993) 63; Nucl. Phys. B399 (1993) 111; H. Murayama and T. Yanagida, Phys. Lett. B322 (1994) 349.

[8] M.Dine, L. Randall, and S. Thomas, hep-ph/9503303 (1995).

[9] R. Barbieri, S. Ferrara, and C.A. Savoy, Phys. Lett. B119 (1982) 343.

[10] G. Dvali, hep-ph/9503259 and hep-ph/9503375 (1995).

[11] G.D. Coughlan, W. Fischler, E.W. Kolb, S. Raby and G.G. Ross, Phys. Lett. B131 (1983) 59.

[12] E. Cremmer, B. Julia, J. Scherk, S. Ferrara, L. Girardello and P. Van Nieuwenhuizen, Phys. Lett. 79B (1978) 231 and Nucl. Phys. B147 (1979) 105; E. Cremmer, S. Ferrara, L. Girardello and A. Van Proeyen, Phys. Lett. 116B (1982) 231 and Nucl. Phys. B212 (1983) 413; R. Arnowitt, A.H. Chamseddine and P. Nath, Phys. Rev. Lett. 49 (1982) 970 and 50 (1983) 232 and Phys. Lett. 121B (1983) 33; J. Bagger and E. Witten, Phys. Lett. 115B (1982) 202 and 118B (1982) 103; J. Bagger, Nucl. Phys. B211 (1983) 302.

[13] J. Polonyi, Budapest preprint KFKI-1977-93.

[14] M.K. Gaillard and V. Jain, Phys. Rev. D49 (1994) 49. (1983) 110. 
[15] P. Binetruy and M.K. Gaillard, Phys. Lett. 195 (1987) 382.

[16] E. Cremmer, S. Ferrara, C. Kounnas and D.V. Nanopoulos, Phys. Lett. 133B (1983) 61.

[17] J. Ellis, A.B. Lahanas, D.V. Nanopoulos and K. Tamvakis, Phys. Lett. 134B (1984) 429; J. Ellis, C. Kounnas and D.V. Nanopoulos, Nucl. Phys. B241 (1984) 406; J. Ellis, C. Kounnas and D.V. Nanopoulos, Nucl. Phys. B247 (1984) 373; for a review see: A.B. Lahanas and D.V. Nanopoulos, Phys. Rep. 145 (1987) 1.

[18] J.D. Breit, B. Ovrut, G. Segré, Phys. Lett. 162B (1985) 303; P. Binétruy and M.K. Gaillard, Phys. Lett. 168B (1986) 347; P. Binétruy, S. Dawson M.K. Gaillard and I. Hinchliffe, Phys. Rev. D37 (1988) 2633 and references therein.

[19] J. Ellis, C. Kounnas and D.V. Nanopoulos, Phys. Lett. 143B (1984) 410.

[20] F. del Aguila, M.K. Gaillard, L. Hall, J. Polchinski, G.G. Ross and B. Zumino, Phys. Lett. 122B (1983) 355.

[21] S. Weinberg, Phys. Rev. Lett. 48 (1982) 1303.

[22] J. Ellis, D.V. Nanopoulos, and M. Quiros, Phys. Lett. B174 (1986) 176.

[23] for a review see: K.A. Olive, Phys. Rep. C190 (1990) 307.

[24] E. Witten, Phys. Lett. 155B: 151 (1985).

[25] S. Ferrara, C. Kounnas and M. Porrati, Phys. Lett. 181B: 263 (1986); L.J. Dixon, V.S. Kaplunovsky and J. Louis, Nucl. Phys. B329: 27 (1990); S. Ferrara, D. Lüst, and S. Theisen, Phys. Lett. 233B: 147 (1989).

[26] G. Gelmini, C. Kounnas, D.V. Nanopoulos, Nucl. Phys. B250 (1985) 177.

[27] P. Binetruy and M.K. Gaillard, Phys. Lett. 232 (1989) 83.

[28] A. Linde, "Particle physics and inflationary cosmology," Harwood Academic, 1990.

[29] H. Murayama, H. Suzuki, T. Yanagida and J. Yokoyama, Phys. Rev. D50 (1994) 2356. 
[30] M.K. Gaillard, V. Jain and K. Saririan, LBL-34948, preprint in preparation.

[31] A.D. Linde, Phys. Lett. 116B (1982) 335; A. Vilenkin and L.H. Ford, Phys. Rev. D26 (1982) 1231; A. Vilenkin, Nucl. Phys. B226 (1983) 527.

[32] R. Holman, P. Ramond, G.G. Ross, Phys. Lett. 137B (1984) 343. 